\title{
HLA Class I Histocompatibility Antigen, A-1 Alpha Chain
}

National Cancer Institute

\section{Source}

National Cancer Institute. HLA Class I Histocompatibility Antigen, A-1 Alpha Chain. NCI

Thesaurus. Code C62772.

HLA class I histocompatibility antigen, A-1 alpha chain protein (365 aa, 41 kDa) is encoded by the HLA-A gene. This protein plays a role in the presentation of foreign antigens to the immune system. It is associated with a number of diseases including graft-versus-host disease, hemochromatosis and certain types of cancer. 